\title{
Trisomy chromosome 5 is a recurrent cytogenetic lesion in mammary tumors from parous MMTV-erbB-2 transgenic mice
}

\author{
YOUNG MI KIM ${ }^{1}$, ZHIKUN MA ${ }^{2}$, SEUNGJIK LEE ${ }^{1}$, JIYUN LEE $^{1}$, SHIBO LI $^{1}$ and XIAOHE YANG ${ }^{2}$ \\ ${ }^{1}$ Department of Pediatrics, University of Oklahoma Health Sciences Center, Oklahoma, OK 73104; \\ ${ }^{2}$ Human Nutrition Program, North Carolina Research Campus, Julius L. Chambers Biomedical/ Biotechnology \\ Research Institute, North Carolina Central University, Kannapolis, NC 28081, USA
}

Received April 19, 2011; Accepted July 1, 2011

DOI: $10.3892 / \mathrm{ol} .2011 .373$

\begin{abstract}
B-2 is amplified or overexpressed in approximately $30 \%$ of human breast cancers, and has been associated with poor prognosis and therapeutic resistance. Previous studies have suggested that erbB-2 overexpression in transgenic mice induces genomic instability; however, the patterns of genetic lesions vary with individual model systems. The development of mammary tumors in multiparous murine mammary tumor virus (MMTV)-erbB-2 transgenic mice is accelerated due to hormonal interactions which induce the overexpression of MMTV-mediated erbB-2. However, whether or not accelerated tumor development is associated with modified cytogenetic patterns remains to be determined. In this study, chromosomal changes were characterized in mammary tumor cells derived from multiparous MMTVerbB-2 transgenic mice, and compared with tumor cells derived from control virgin mice. Immunohistochemistry and Western blotting were used to detect erbB-2 overexpression in mammary tissues. Each of the five tumors from the multiparous MMTV-erbB-2 transgenic mice was found to exhibit a marked chromosomal imbalance, compared with only one tumor with aberrant chromosomes among the five tumors from the control virgin mice. In particular, trisomy 5 and loss of the $\mathrm{X}$ chromosome were recurrent cytogenetic lesions in tumors from the parous mice, which is a novel pattern compared with previous studies. The elevated number of genetic lesions in tumors from parous mice, which were characterized by enhanced erbB-2 overexpression and increased receptor tyrosine kinase activation in the mammary glands, suggest a causal role for erbB-2
\end{abstract}

Correspondence to: Dr Shibo Li, Department of Pediatrics, University of Oklahoma Health Sciences Center, Oklahoma, OK 73104, USA

E-mail: shibo-li@ouhsc.edu

Dr Xiaohe Yang, Human Nutrition Program, North Carolina Research Campus, Julius L. Chambers Biomedical/ Biotechnology Research Institute, North Carolina Central University, Kannapolis, NC 28081, USA

E-mail: xyang@nccu.edu

Key words: erbB-2/Neu, genomic instability, breast cancer, transgenic mouse model in the genomic instability present in these tumors. These data advance our understanding of erbB-2-mediated pathogenesis and underscore the role of cytogenetic alteration in this process.

\section{Introduction}

erbB-2 (HER2, Neu) is a receptor tyrosine kinase of the EGFR family $(1,2)$. erbB-2 is amplified/overexpressed in approximately $30 \%$ of primary human breast cancers, and has been associated with poor prognosis and therapeutic resistance $(3,4)$. erbB-2 overexpression and/or activation induces the subsequent activation of a plethora of signaling pathways, including those that are mediated by MAP kinase, PI3 kinase, and the STAT family of transcription factors $(5,6)$. These activated signaling pathways ultimately increase cell proliferation, reduce apoptosis, and induce cell transformation (7). erbB-2-associated carcinogenesis has been extensively studied. However, since erbB-2 activation elicits signaling in diversified downstream pathways, the precise mechanisms involved in erbB-2-mediated carcinogenesis remain unclear.

erbB-2 transgenic mouse models were utilized in numerous studies in order to understand erbB-2-mediated carcinogenesis (8). The association of erbB-2 overexpression with genomic instability is of marked interest. Montagna et al reported that tumor cell lines from transgenic mice overexpressing constitutively activated mutant erbB-2/Neu exhibited recurrent deletions of chromosome 4, amplification of chromosome 11, and abnormalities in the centrosome (9). In previous studies, we detected a number of cytogenetic lesions using mammary tumor cell lines derived from wild-type (wt) MMTV-erbB-2/ Neu transgenic mice. The most common chromosomal abnormalities were loss of mouse chromosome 4 and gain of chromosome 10 (10). We also revealed that tumors and tumor cell lines derived from MMTV-erbB-2 mice treated with E2 or soy appeared to have more cytogenetic lesions (10). These results suggest that the chromosomal imbalance in the erbB-2-associated cytogenetic changes are affected by erbB-2 signaling activity (mutant or activated erbB-2 induces stronger carcinogenic activity) and hormonal conditions.

Although previous studies suggest a correlation between erbB-2 overexpression and genomic instability, and since the patterns of cytogenetic changes vary with individual model 
systems, the effects of erbB-2 overexpression on genomic instability in mammary tumor development require further investigation. The purpose of the present study was to examine the cytogenetic patterns in mammary tumor cells derived from multiparous and virgin control wt MMTV-erbB-2 mice. Since the MMTV promoter is sensitive to pregnancy hormones such as prolactin, multiparity enhances MMTV-mediated transcription (11), and parous MMTV-erbB-2 mice usually exhibit accelerated mammary tumor development due to the enhanced overexpression of MMTV-erbB-2 during pregnancy and lactation (12). Therefore, this model system allowed us to examine the effects of erbB-2 overexpression and increased activation on chromosomal imbalance.

\section{Materials and methods}

Animals and tumor samples. The animals used in this study were MMTV-erbB-2 mice (wt erbB-2 mice) from Jackson Laboratory (Bar Harbour, ME, USA). Following a protocol approved by the university's Institutional Animal Care and Use Committee (IACUC), the mice were fed a lifelong AIN-93G diet and housed in the barrier facility at the University of Oklahoma Health Sciences Center. Mammary tumors developed from control virgin MMTV-erbB-2 mice and from mice with three full-term pregnancies. The tumors were harvested once they reached $1 \mathrm{~cm}^{3}$ in diameter. Five primary tumors from five different mice in each group were collected. For the samples used to evaluate erbB-2 expression, mammary tissues were obtained from control virgin mice and from the parous mice one day after parturition in the third pregnancy (at 24 weeks).

Western blot analysis. Cell lysates were prepared from the mammary tissues of virgin control and parous mice one day after parturition. Protein lysates $(50 \mu \mathrm{g})$ from each sample were separated using a $10 \%$ SDS-PAGE gel and transferred to nitrocellulose membrane. The membrane was probed with antibodies against erbB-2 and actin (Santa Cruz Biotechnology, Santa Cruz, CA, USA). Following incubation with secondary antibodies and subsequent washing, the specific bands were visualized with an ECL kit (Thermo Fisher Scientific, Miami, OK, USA).

Immunohistochemistry. Immunohistochemistry was performed as previously reported (13). Briefly, mammary tissues from virgin control or parous mice were fixed in formaldehyde. Tissue sections were deparaffinized and rehydrated. Non-specific binding sites were blocked with $10 \%$ normal horse serum and incubated overnight with anti-erbB-2 monoclonal antibody. Following incubation with biotinylated goat anti-mouse antibody, the signals were visualized using the ABC kit (Vector Lab, Burlingame, CA, USA).

Primary cell culture and sample preparation. Tumors were aseptically removed from the animals and immediately processed during the tumor harvest $(10,13)$. Tumor tissues were minced with scissors and then washed with PBS. The tissue explants were cultured in a flask containing DMEM/ F12 media supplemented with $10 \%$ FBS and penicillin/ streptomycin. Outgrowth from the explants was trypsinized and isolated as primary cell lines. After removing the explants, cells in the second passage were used for cytogenetic analysis.

Chromosome preparation and karyotype analysis. Primary mammary tumor cells were arrested in metaphase by adding colcemid (final concentration of $0.02 \mu \mathrm{g} / \mathrm{ml}$ ) (Gibco, Carlsbad, CA, USA) to the culture media for $1 \mathrm{~h}$. The cells were harvested according to the standard protocols in our laboratory. Chromosomes were treated and stained using trypsin-Giemsa banding (GTG-banding). At least 15 metaphase cells were analyzed and karyotyped from each cell line.

Fluorescent in situ hybridization (FISH). The whole chromosome painting (WCP) probe for chromosome 5 was purchased from commercial sources (Cambio). FISH was performed according to the manufacturer's instructions. Twenty metaphase cells were analyzed for each cell line.

\section{Results}

Enhanced overexpression of erbB-2 in parous mammary tissues. To assess the effect of pregnancy on MMTV-induced erbB-2 expression, the expression of erbB-2 was examined in mammary tissues from MMTV-erbB-2 mice one day after parturition in the third pregnancy, and compared to that of control virgin mice at the same age (24 weeks). Fig. 1A shows that erbB-2 protein levels in the parous mammary tissues were markedly higher than those in the control. The immunohistochemical examination indicated that erbB-2 was overexpressed in the luminal mammary epithelial cells. In contrast to the virgin mice, enhanced overexpression of erbB-2 was detected in all of the alveolar epithelial cells of the newly parturated mice (Fig. 1B). The increased erbB-2 levels and the percentage of positive cells in the parous mammary tissues may contribute to the cytogenetic alterations detected in the subsequent experiments.

Cytogenetic alterations in mammary tumor cells from control and parous MMTV-erbB-2 transgenic mice. To determine whether the mammary tumors in parous MMTV-erbB-2 mice acquired more cytogenetic lesions, the chromosomal changes were characterized in primary mammary tumor cells derived from control virgin MMTV-erbB-2 mice and parous mice with three full-term pregnancies. In each group, five tumor cell lines from five different mice were analyzed using G-banded karyotyping. As shown in Table I, four of the five cell lines from the virgin control mice had a 'normal' karyotype. One of these cell lines (VMT-3) contained a chromosome 4 deletion and trisomy 5. In contrast, each of the tumor cell lines from the parous mice exhibited significant chromosomal changes, indicating that the tumors developing from parous mice acquired more genetic lesions. Specifically, trisomy 5 (Fig. 2B) was detected in 3 of the 5 cell lines (PMT-3, 4 and 5); the deletion of chromosome 4 (Fig. 2C) was detected in 2 of the 5 cell lines (PMT-1 and 2); and the loss of chromosome X (Fig. 2D) was detected in 2 of the 5 cell lines (PMT-2 and 5). In the context of previous studies on cytogenetic changes in mammary tumors induced by the overexpression of mutant or wt erbB-2 $(9,10)$, we have identified trisomy 5 as a previously unidentified characteristic change in tumors from parous mice. 
Table I. Karyotypes of primary mammary tumor cells derived from virgin and parous MMTV-erbB-2 transgenic mice.

\begin{tabular}{lll}
\hline Origin & Cell lines & \multicolumn{1}{c}{ Karyotype } \\
\hline Control virgin mouse tumors & VMT-1 & $40, \mathrm{XX}$ \\
& VMT-2 & $40, \mathrm{XX}$ \\
& VMT-3 & $40, \mathrm{XX}, \mathrm{add}(4)(\mathrm{E} 2)[3] / 41, \mathrm{XX},+5[2] / 40, \mathrm{XX}[15]$ \\
& VMT-4 & $40, \mathrm{XX}$ \\
Parous mouse tumors & VMT-5 & $40, \mathrm{XX}$ \\
& PMT-1 & $40, \mathrm{XX}, \operatorname{del}(4)(\mathrm{C} 2 \mathrm{D} 1)[3] / 40, \mathrm{XX}[12]$ \\
& PMT-2 & $39, \mathrm{X},-\mathrm{X}[14] / 40, \mathrm{XX}, \mathrm{del}(4)(\mathrm{C} 2 \mathrm{D} 1)[1]$ \\
& PMT-3 & $41, \mathrm{XX},+5[9] / 40, \mathrm{XX}[7]$ \\
& PMT-4 & $41, \mathrm{XX},+5[6] / 40 . \mathrm{XX}[9]$ \\
& PMT-5 & $41, \mathrm{XX},+5[2] / 39, \mathrm{X},-\mathrm{X}[2] / 40 . \mathrm{XX}[14]$ \\
\hline
\end{tabular}

A

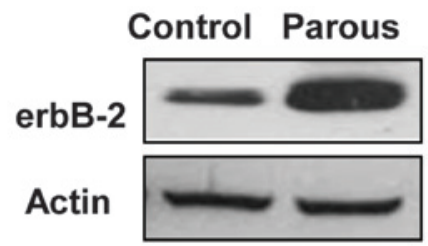

$\mathbf{B}$

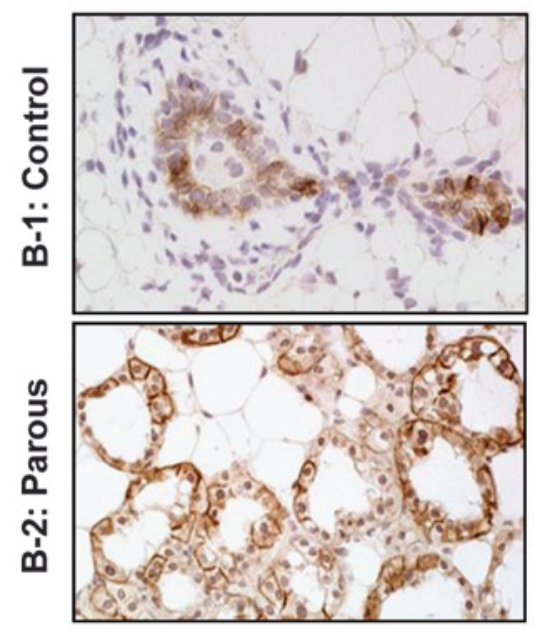

Figure 1. The expression of erbB-2 in mammary tissues from control and parous MMTV-erbB-2 transgenic mice. The protein lysates and mammary tissue blocks from the parous mice were prepared one day after parturition in the third pregnancy (at 24 weeks). Controls were virgin MMTV-erbB-2 mice (also 24 weeks old). Relative erbB-2 levels in mammary tissues were detected by (A) Western blot analysis erbB-2 expression was detected by (B) immunohistochemistry (magnification, $\mathrm{x} 400$ ).

Trisomy 5 confirmation by FISH. The chromosomal status in the tumor cell lines was analyzed by FISH using a whole chromosome painting probe for chromosome 5. Consistent with the karyotyping data, trisomy 5 detected using G-banding was confirmed by FISH in the corresponding mammary tumor cells (Fig. 3).

\section{Discussion}

Although carcinogenesis in erbB-2 transgenic mice is mainly driven by erbB-2 overexpression, accumulating data suggest that the tumor development in these mice involves the acquisition of additional genetic defects. For example, the p53 mutation is a common target of additional genetic defects that facilitate erbB-2-mediated carcinogenesis (14). Breeding mice that express mutant 553 (p53-172H) with MMTV-erbB-2 transgenic mice causes accelerated tumor development $(14,15)$. These results suggest that tumors exhibiting a chromosomal imbalance play a critical role in erbB-2-mediated carcinogenesis.

Previous studies from our group and other authors indicate that erbB-2-mediated tumorigenesis in erbB-2 transgenic mouse models involves a chromosomal imbalance $(9,10)$. However, it remains unclear whether or not these cytogenetic changes were complementary to erbB-2 overexpression or were caused by erbB-2 overexpression and increased activation. This study aimed to characterize chromosomal changes in mammary tumors that developed in wt MMTV-erbB-2 transgenic mice with multiparity and to test whether enhanced overexpression of erbB-2 and hormonal modulation in these mice induced chromosomal changes. The results demonstrated that all tumors from the parous mice exhibited a marked increase in cytogenetic lesions, compared with only one of the five tumors from virgin mice that contained aberrant chromosomes. Since tumor development in parous mice involves hormonal fluctuations that enhance erbB-2 overexpression and activation, the increase in the number of genetic lesions in the parous group appears to be caused by the enhanced erbB-2 expression and activation. Recurrent changes from mice with the same transgenic background but varying erbB-2 levels support the causal role of erbB-2 overexpression in the induction of genomic instability.

The karyotype analysis also revealed that the chromosomal imbalance in these tumors involves the recurrent trisomy chromosome 5 ( 3 of the 5 cell lines), the deletion of the entire $\mathrm{X}$ chromosome ( 2 of the 5 cell lines), and the partial deletion of chromosome 4 ( 2 of the 5 cell lines), suggesting a new pattern that has not previously been characterized in other studies. Previous studies that have analyzed transgenic mice overexpressing mutant or constitutively activated erbB-2 have shown that the most frequent cytogenetic alterations were deletions in chromosome 4 and gains in chromosome 11. Trisomy chromosome 5 was only detected in one of the 22 tumors examined (9). In our previous study, using the virgin wt MMTV-erbB-2 transgenic model, partial or whole chromosome 4 deletion was 
A

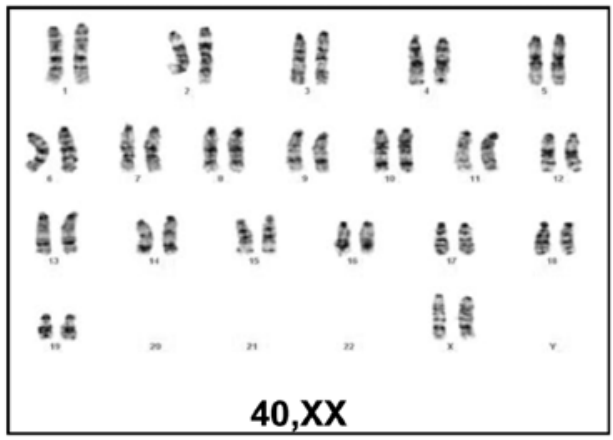

C

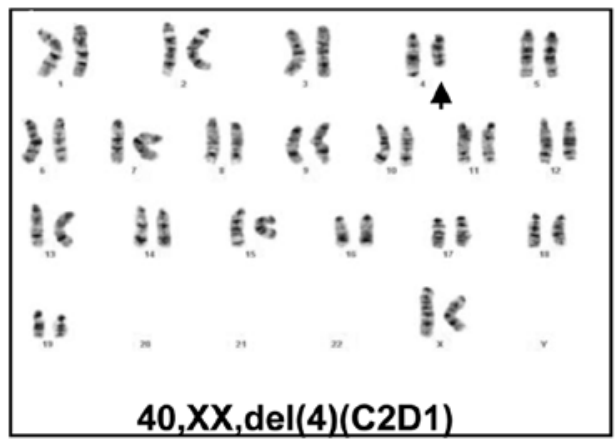

B

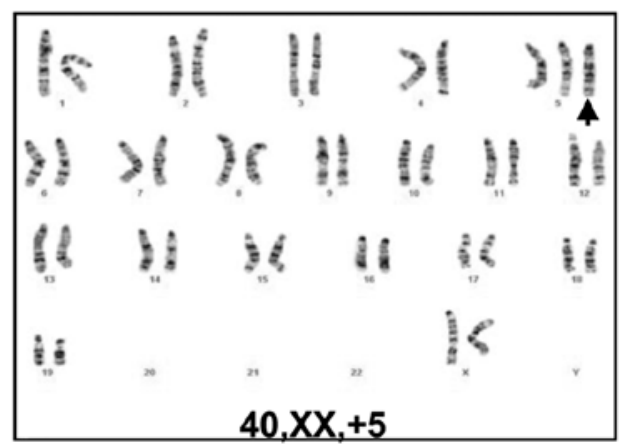

D

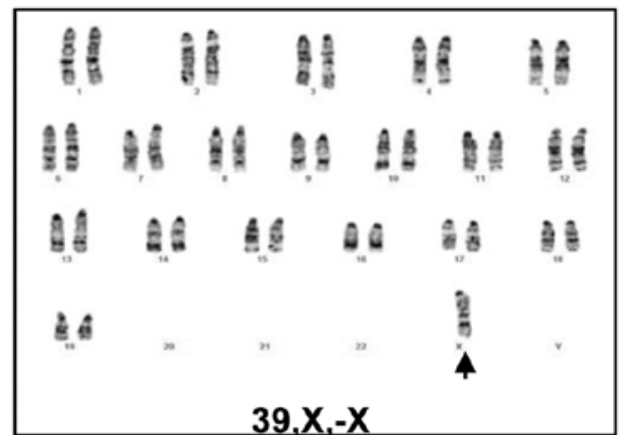

Figure 2. GTG-banded karyotype of mammary tumor cell lines with (A) normal and altered (B, C and D) karyotypes. (B) Trisomy 5 was detected in 3 of the 5 cell lines (PMT-3, 4 and 5). (C) Deletion of chromosome 4 at band C2D1 was detected in 2 of the 5 cell lines (PMT-1 and 2). (D) Loss of the X chromosome was detected in 2 of the 5 cell lines (PMT-2 and 5).

A

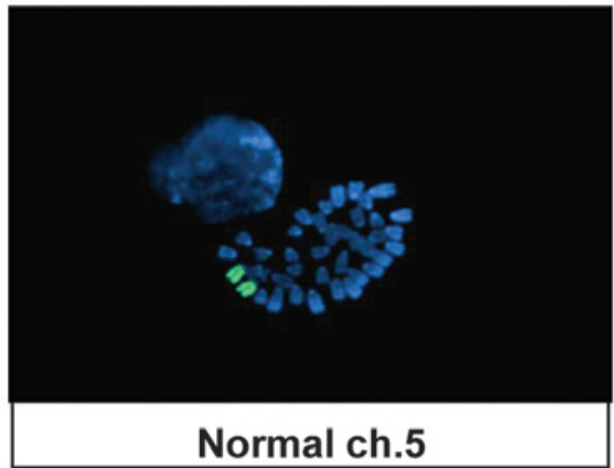

B

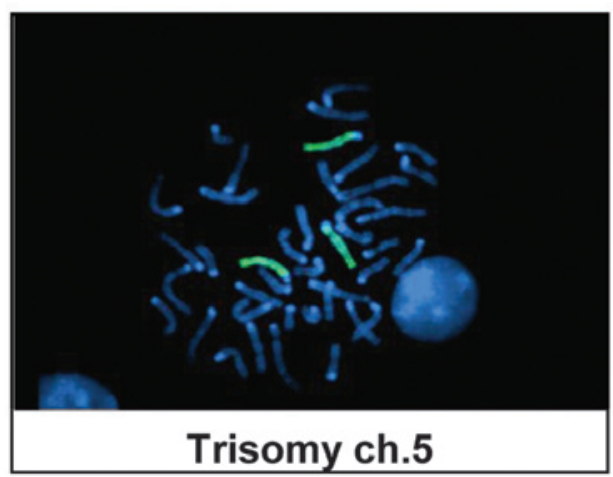

Figure 3. FISH analysis using the WCP probe for chromosome 5 (green) in mammary tumor cells (PMT-3). FISH results show trisomy chromosome 5; ch., chromosome.

common but trisomy chromosome 5 was not detected (10). In the context of these studies, our current results suggest that cytogenetic changes in tumors from parous mice, not only occur at a higher frequency, but also indicate a pattern that is inconsistent with that reported in previous studies. Trisomy 5 and loss of $\mathrm{X}$ chromosome appear to be associated with enhanced overexpression and activation of erbB-2.

Notably, although the chromosome 4 deletion was a common lesion detected in previous studies $(9,10)$, this deletion is not the most common change ( 2 in 5 cell lines) evident in the current study. Nevertheless, repeated detection of chromosome 4 deletion in various studies underscores its significance in erbB-2-mediated genomic instability. Previous CGH analysis indicated that the region frequently lost in mouse chromosome 4 is mapped to human chromosome 1p35-36, which contains potential tumor suppressors, including 14-3-30. Of note is that deficiency of 14-3-30 in numerous primary human breast cancers has been attributed to loss of the chromosome 1p35-36 (16). Further comparative studies of erbB-2-associated cytogenetic changes between mouse and human models may clarify this correlation. Results from this study support further investigation into the effect of enhanced overexpression of erbB-2 on genomic instability and the role of cytogenetic factors in erbB-2-mediated breast cancer development. Although changes at the chromosomal level cannot reflect micro-level lesions, the data in this study are fundamental for future examinations with novel approaches, including a CGH microarray.

In conclusion, the results from this study indicate that tumors from multiparous mice contain more chromosomal aberrations. The study also demonstrates that trisomy chromosome 5 is a recurrent cytogenetic lesion in mammary tumors from multiparous MMTV-erbB-2 transgenic mice. These results identify 
a new pattern of cytogenetic lesions that may contribute to erbB-2-mediated carcinogenesis. Moreover, multiparity is known to be associated with erbB-2-mediated carcinogenesis in human breast cancer; however, the underlying mechanisms have yet to be elucidated. Further characterization of cytogenetic changes associated with erbB-2 overexpression, and hormonal modulation are likely to be invaluable in directing the focus of clinical studies.

\section{Acknowledgements}

X. Yang and Z. Ma were supported in part by a Research Scholar Grant from the American Cancer Society (RSG-08-138-01-CNE) and by the Oklahoma Center for the Advancement of Science and Technology.

\section{References}

1. Bargmann CI, Hung MC and Weinberg RA: The neu oncogene encodes an epidermal growth factor receptor-related protein. Nature 319: 226-230, 1986.

2. Schechter AL, Stern DF, Vaidyanathan L, et al: The neu oncogene: an erb-B-related gene encoding a 185,000-Mr tumour antigen. Nature 312: 513-516, 1984.

3. Koeplinger KA, Mildner AM, Leone JW, et al: Caspase 8: an efficient method for large-scale autoactivation of recombinant procaspase 8 by matrix adsorption and characterization of the active enzyme. Protein Expr Purif 18: 378-387, 2000.

4. Slamon DJ, Clark GM, Wong SG, et al: Human breast cancer: correlation of relapse and survival with amplification of the HER-2/neu oncogene. Science 235: 177-182, 1987.

5. Stern DF: Tyrosine kinase signalling in breast cancer: ErbB family receptor tyrosine kinases. Breast Cancer Res 2: 176-183, 2000 .
6. Prenzel N, Fischer OM, Streit S, et al: The epidermal growth factor receptor family as a central element for cellular signal transduction and diversification. Endocr Relat Cancer 8: 11-31, 2001.

7. Muthuswamy SK, Gilman M and Brugge JS: Controlled dimerization of ErbB receptors provides evidence for differential signaling by homo- and heterodimers. Mol Cell Biol 19: 6845-6857, 1999.

8. Siegel PM, Ryan ED, Cardiff RD, et al: Elevated expression of activated forms of Neu/ErbB-2 and ErbB-3 are involved in the induction of mammary tumors in transgenic mice: implications for human breast cancer. Embo J 18: 2149-2164, 1999.

9. Montagna C, Andrechek ER, Padilla-Nash H, et al: Centrosome abnormalities, recurring deletions of chromosome 4 , and genomic amplification of HER2/neu define mouse mammary gland adenocarcinomas induced by mutant HER2/neu. Oncogene 21: 890-898, 2002

10. Jeruss JS, Liu NX, Chung Y, et al: Characterization and chromosomal instability of novel derived cell lines from a wt-erbB-2 transgenic mouse model. Carcinogenesis 24: 659-664, 2003.

11. Wagner KU, McAllister K, Ward T, et al: Spatial and temporal expression of the Cre gene under the control of the MMTV-LTR in different lines of transgenic mice. Transgenic Res 10: 545-553, 2001.

12. Anisimov VN, Popovich IG, Alimova IN, et al: Number of pregnancies and ovariectomy modify mammary carcinoma development in transgenic HER-2/neu female mice. Cancer Lett 193: 49-55, 2003.

13. Kim A, Liu B, Ordonez-Ercan D, et al: Functional interaction between mouse erbB3 and wild-type rat c-neu in transgenic mouse mammary tumor cells. Breast Cancer Res 7: R708-R718, 2005.

14. Li B, Rosen JM, McMenamin-Balano J, et al: Neu/ERBB2 cooperates with p53-172H during mammary tumorigenesis in transgenic mice. Mol Cell Biol 17: 3155-3163, 1997.

15. Brodie SG, Xu X, Li C, et al: Inactivation of $\mathrm{p} 53$ tumor suppressor gene acts synergistically with c-neu oncogene in salivary gland tumorigenesis. Oncogene 20: 1445-1454, 2001.

16. Hodgson JG, Malek T, Bornstein S, et al: Copy number aberrations in mouse breast tumors reveal loci and genes important in tumorigenic receptor tyrosine kinase signaling. Cancer Res 65: 9695-9704, 2005. 